\title{
Pasteurella multocida infections in cancer patients
}

\author{
Marela Vélez ${ }^{\mathrm{a}}$, Beata Casanasa, John N Greene ${ }^{\mathrm{b}}$, Jose Morey ${ }^{\mathrm{a}}$, Dominick Mastroianni ${ }^{\mathrm{a}}$, Richard Oehler ${ }^{\mathrm{a}}$ \\ ${ }^{a}$ Division of Infectious Diseases and International Medicine, University of South Florida College of \\ Medicine, Tampa, FL 33606; ' Moffitt Cancer Center and Research Institute, University of South \\ Florida College of Medicine, Tampa, Florida 33612-9497, USA
}

\begin{abstract}
Background: Pasteurella multocida is a small, gram-negative coccobacillus, which most commonly causes soft tissue infections due to animal bites or scratches, mainly from cats and dogs. Immunocompromised hosts, such as cancer patients, are more likely to develop systemic complications as a result of $P$. multocida infections.

Objective: Retrospectively analyze the medical records of four afflicted patients being managed at Moffitt Cancer Center, Tampa, USA between 1999 and 2009, and careful study for additional 32 cases of P. multocida infection among cancer patients with variegated histology found in the current medical literature.

Methods: Of 36 subjects, $67 \%$ of the patients had been diagnosed with a solid organ cancer, whereas $33 \%$ had a hematologic malignancy. Clinical scenarios described fever as the most frequent initial presentation and bacteremia as the most prevalent mode of infection.

Results: Forty-seven percent of the patients had experienced some sort of animal contact and $41 \%$ showed evidence of skin or soft tissue infection. The status of the white blood cell count, was available in 22 patients (of 36 patients), and $27 \%$ demonstrated neutropenia. The survival percentage of the patients with known clinical outcome was $77 \%$.

Conclusion: Medical management mostly involved antibiosis with beta-lactams. Atypical scenario of Pasteurella multocida infection may involve bites or scratches (specifically from cats or dogs) in a cancer patient presenting with sepsis and accompanied by skin or soft tissue or respiratory tract infection. A high level of suspicion for P. multocida as a possible pathogen in cancer patients would facilitate an amelioration in morbidity ameliorating, and timely initiation of specific antibiotics.
\end{abstract}

Keywords: Beta-lactams, infections, malignancy, Pasteurella multocida

Pasteurella multocida is a small, non-motile, nonspore forming, gram-negative coccobacillus, which is found in the oral and nasal secretions of a large variety of household pets such as cats and dogs and barnyard animals including cattle, horses, swine and sheep, as well as rabbits and mice. However, since the highest carriage rate occurs in cats (70 to $90 \%$ ) and dogs $(66 \%)$, infections in humans generally occur as a result of cat bites or scratches and dog bites [1]. $P$. multocida causes a wide array of presentations, the most common being cutaneous, or soft tissue infections, followed by infections of the lower

Correspondence to: Professor John N. Greene, Moffitt Cancer Center and Research Institute, USFCOM, Magnolia Drive, FOB-3, Tampa, FL33612-9497, USA. E-mail: John.Greene@ moffitt.org respiratory tract, such as pneumonia, empyema and lung abscess. Systemic infections, such as bacteremia, meningitis or peritonitis are also common presentations [2]. Immunocompromised hosts, particularly cancer patients, are more likely to develop the more severe, systemic presentations as a result of $P$. multocida infections [3]. In this report, we described four such cases from the Moffitt Cancer Center in Tampa, USA, and review 32 other previously-reported cases found in the medical literature.

\section{Methods}

An epidemiology report generated at Moffitt Cancer Center between January 1999 and April 2009 identified four cases of $P$. multocida infections. The medical records of the four affected patients were 
retrospectively analyzed for age, sex, underlying malignancy, animal contact, initial presentation, diagnostic culture source, evidence of neutropenia (ANC $<0.5 \times 10^{9} / \mathrm{L}$ ). Treatment and outcome were also considered. In addition, we undertook a rigorous search of the current medical literature for cases of P. multocida infection among cancer patients, focusing on the preceding parameters. We summarize the data in Table 1.

\section{Results}

Among the 36 patients with Pasteurella infection, $58 \%$ were females and $42 \%$ were males. The patients varied in age from 18 to 87 years with a median of 64 years. Sixty-seven percent of the patients had a solid-organ malignancy, and 33\% had a hematogenous malignancy. Out of the 36 patients, a history of animal contact was verified in only 19 patients and from that subgroup, most patients (89\%) were exposed to a cat or dog. Initial clinical presentation was identified in 27 patients and fever was the most common presenting sign $(78 \%)$ followed by soft tissue involvement (59\%), and altered mental status (19\%). Diagnosis was made by culture of different specimens, including blood (18 patients or 50\%), lower respiratory tract (nine patients or $25 \%$ ), and skin, soft tissue, and joint fluid (eight patients or 31\%). Rare sites of infection included the female genital tract (two patients) and the central nervous system (two patients). Treatment was described in 25 patients and 23 of those received beta-lactam therapy, including penicillins and cephalosporins. Thirteen patients (52\%) were treated with penicillins (including natural penicillin, ampicillin, amoxicillin, and piperacillin) and 10 patients $(40 \%)$ received some type of cephalosporin, cefepime and ceftazidime. The white blood cell count was available in 22 patients out of the 36 patients. In $27 \%$ of patients, it was consistent with neutropenia. Although clinical outcome was unknown in ten patients, the survival and mortality rate of the rest of the patients was $77 \%$ and $23 \%$, respectively. Out of the six patients that died, five where diagnosed with bacteremia.

\section{Discussion}

P. multocida is a small, non-motile, non-spore forming, aerobic and facultative anaerobic gramnegative coccobacillus. This bacteria grows on blood, chocolate, and Mueller-Hinton agar at 37 degrees, but it will not grow on MacConkey's medium $[1,3]$.
Seventy to ninety percent of cats and 50 to $60 \%$ of dogs carry P. multocida in their oral or nasal secretions. This organism is also found in the respiratory and digestive tract of horses, cattle, sheep, rabbits and swine as well as wild animals such as rats, mice, reindeer, and monkeys, and in large mammals like buffaloes, lions, and panthers [1, 4]. Kimura et al. [5] proposed that transmission of Pasteurella to humans involves close contact with the animal's oral cavity. Therefore, the most common portal of entry of P. multocida in humans is through local wound infection after direct animal bites or a scratch, which can deposit the bacteria directly on damaged skin, or after a pet lick on an exposed human mucosal surface [6].

Infections due to $P$. multocida in a normal population have been categorized as: 1) a local infection following an animal bite or scratch that can progress to variegated presentations such as abscess formation, tenosynovitis, septic arthritis and osteomyelitis, 2) an infection of the respiratory tract (pneumonia, empyema and lung abscess), and 3) a systemic infection, for instance, intra-abdominal infections, peritonitis, meningitis and brain abscess $[2,7,8]$.

Specific settings such as in elderly patients, and especially those with associated immunosuppressed states from chronic disease or cancer, are at higher risk of more complicated severe infections. The most common chronic medical condition predisposing to P. multocida infections is liver cirrhosis [3, 4]. As a corollary $P$. multocida bacteremia is uncommon in the general population, it is diagnosed mostly in immunocompromised populations, mainly in cirrhotics and cancer patients [6]. Similarly, this review, encompassed a substantial number of immunodeficient patients, and unsurprisingly, demonstrated that half of the patients had been diagnosed with bacteremia.

Weber et al. [1] analyzed various $P$. multocida isolates for in vitro antibiotic susceptibility and noted that, in general, the antibiotics with the best activity were penicillin $\mathrm{G}$, penicillin $\mathrm{V}$, ampicillin, carbenicillin, ticarcillin, piperacillin and mezlocillin. In addition, second and third generation cephalosporins, tetracyclines and chloramphenicol were also effective. It is worth noting that fluoroquinolones (ciprofloxacin and levofloxacin) are endowed with in vitro activity.

Nevertheless, penicillin is the drug of choice to treat $P$. multocida infections in the non-allergic population. However, Jordan et al. [9] reported a case 


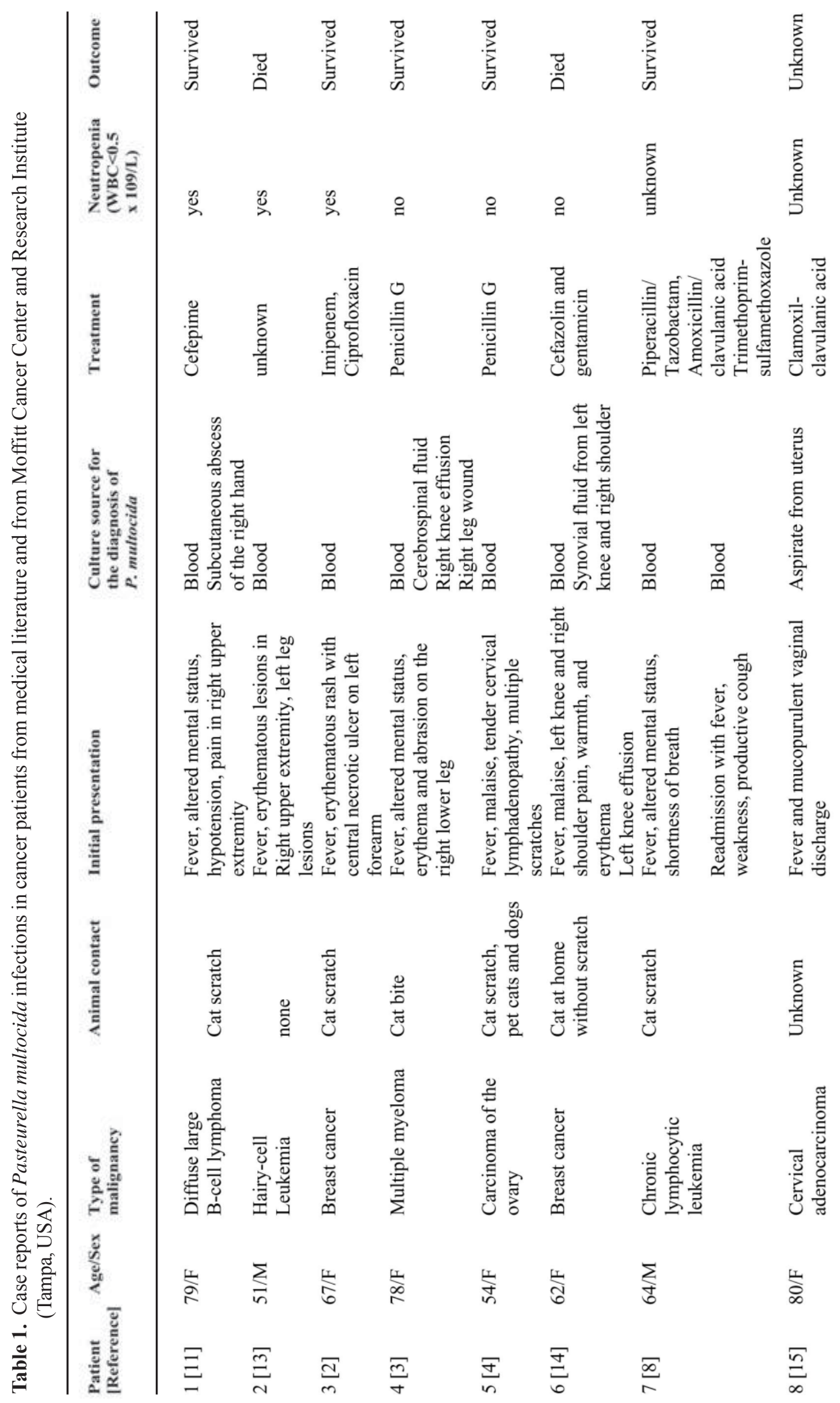




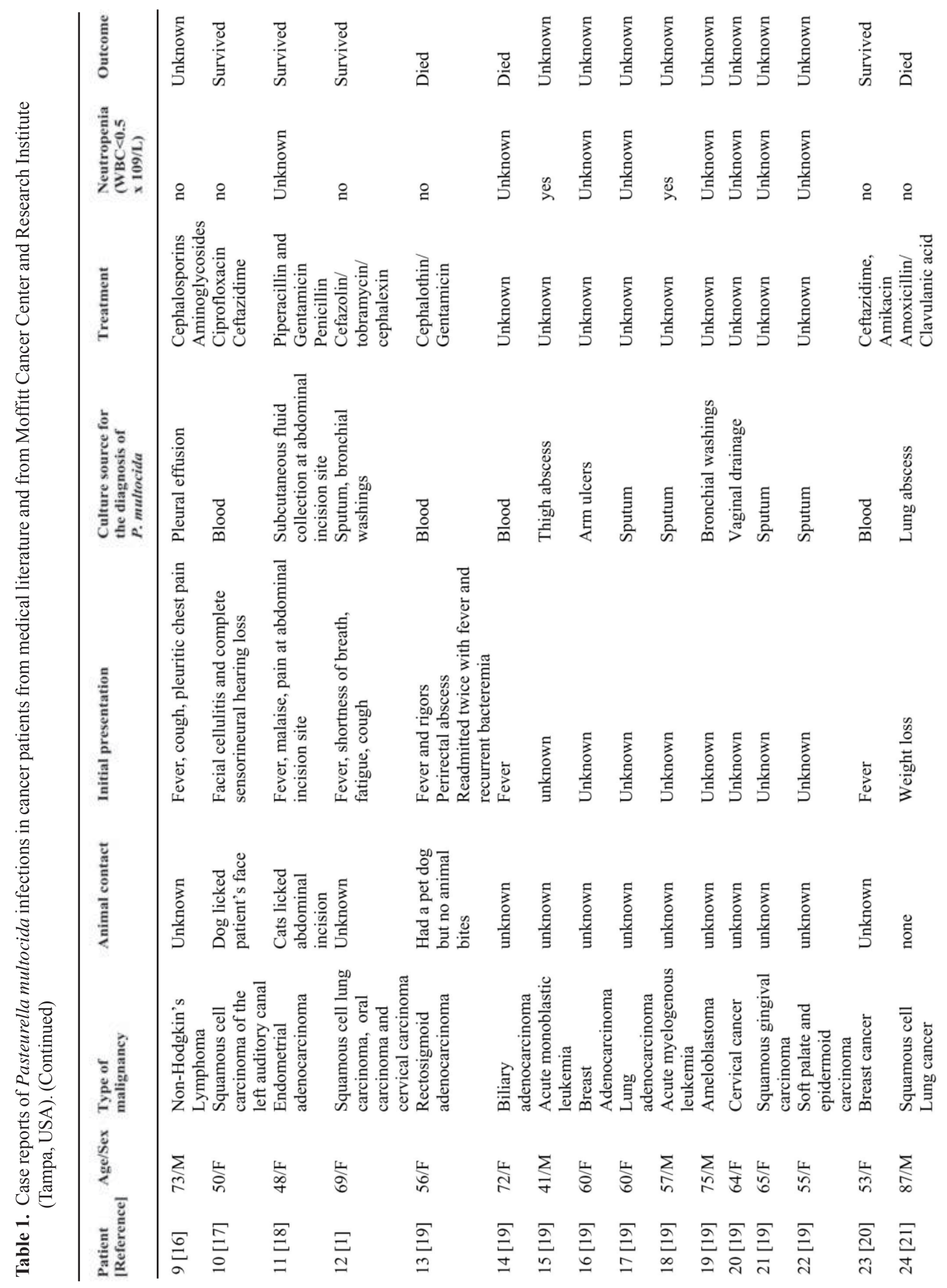




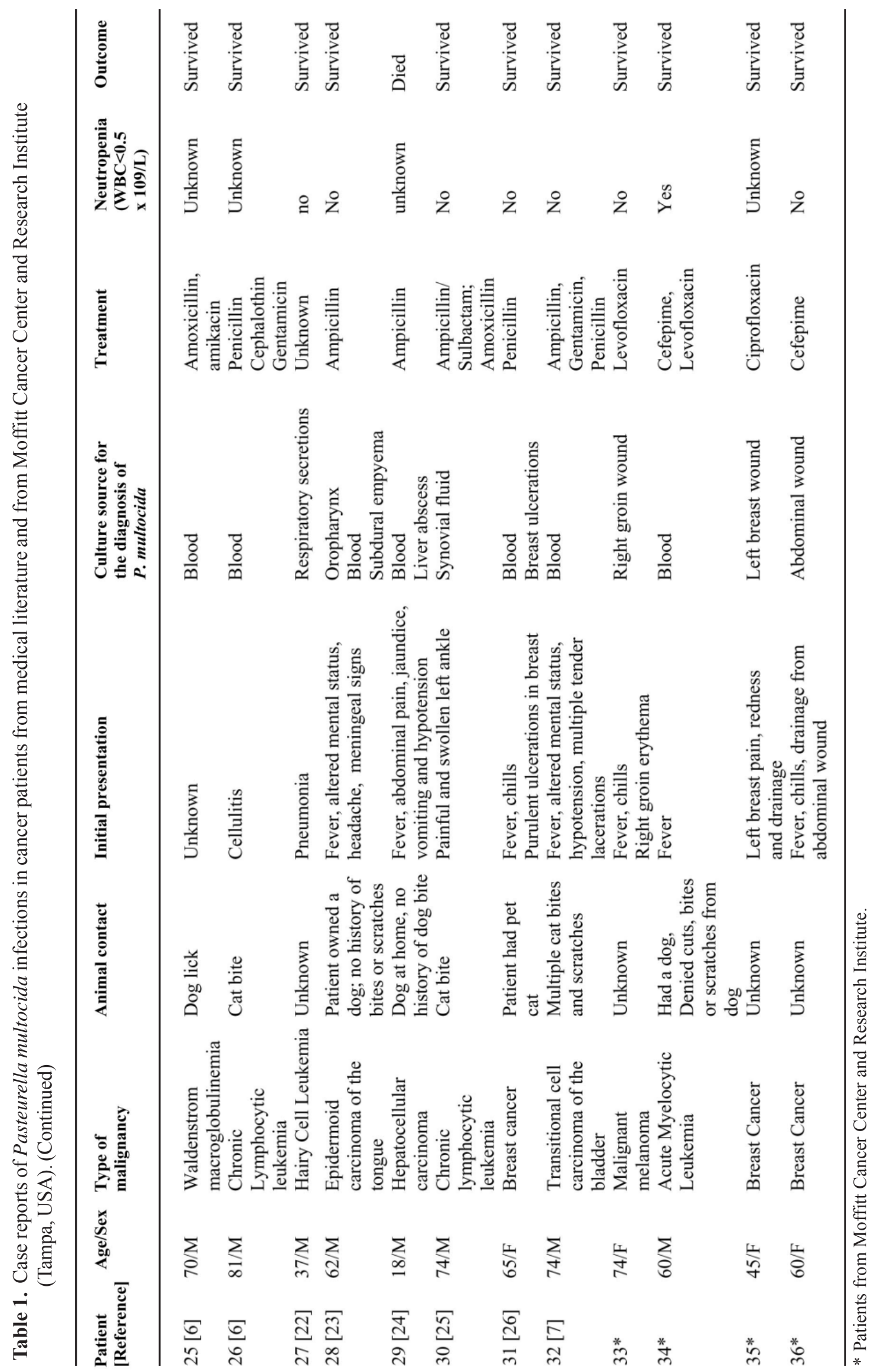


of $P$. multocida meningitis treated effectively with aztreonam. It is worth emphasizing however, that aminoglycosides, vancomycin, erythromycin, and clindamycin should be avoided in the penicillin-allergic patient since drug resistance may result [3]. In addition, when considering empiric antibiotic therapy for an animal bite wound, dicloxacillin, erythromycin, and clindamycin should be avoided as well as cephalosporins such as cephalexin, cefaclor and cefadroxil, since $P$. multocida isolates have been shown to be resistant to all of these antibiotics [10]. Finally, Yokose et al. [11] described a severely neutropenic cancer patient with $P$. multocida bacteremia and sepsis who was successfully treated with cefepime monotherapy.

In summary, P. multocida should be considered as a possible etiologic agent in any patient with a local wound infection secondary to an animal bite or scratch. This pathogen is recognized as opportunistic, and it should always be included in the differential diagnosis of any immunocompromised patient with bacteremia and a history of an animal bite wound and/or close animal contact [6]. Post-chemotherapy neutropenic patients or anyone afflicted with severe immunosuppression should avoid close or accidental contact with pet or barnyard animals [11].

Among our study patients, vital epidemiological information, such as animal contact, either may have been either absent or was inadequately documented in some of the cases, which may represent a methodological flaw. Nevertheless, cases of P. multocida bacteremia lacking history of animal exposure have been repeatedly described; perhaps simply representing previous asymptomatic colonization of the respiratory or gastrointestinal tract in those afflicted [12]. Furthermore, patients presenting with Pasteurella sepsis without a documented animal exposure should undergo extensive evaluation in order to exclude the presence of an underlying immunodeficiency [13].

The authors have no conflict of interest to report.

\section{References}

1. Weber DJ, Wolfson JS, Swartz MN, Hooper DC. Pasteurella multocida infections - report of 34 cases and review of the literature. Medicine. 1984; 63:133-54.

2. Gowda RV, Stout R. Pasteurella multocida infection in a post-chemotherapy neutropenic host following cat exposure. Clinical Oncology 2002; 14:497-8.
3. Layton CT. Pasteurella multocida meningitis and septic arthritis secondary to a cat bite. J Emerg Med. 1999; 17:445-8.

4. Casey AC, Greenspoon JS, Lagasse LD. Pasteurella multocida bacteremia in a patient with ovarian cancer and chemotherapy-induced neutropenia. Infect Dis Obstet Gynecol. 1995; 3:205-9.

5. Kimura R, Hayashi Y, Takeuchi T, Shimizu M, Iwata M, Tanahashi J, Ito M. Pasteurella multocida septicemia caused by close contact with a domestic cat: case report and literature review. J Infect Chemother. 2004; 10:250-2.

6. Raffi F, Barrier J, Baron D, Drugeon HB, Nicolas F, Courtieu AL. Pasteurella multocida bacteremia- report of 13 cases over 12 years and review of the literature. Scand J Infecti Dis. 1987; 19:385-93.

7. Morris MJT, McAllister CK. Bacteremia due to Pasteurella multocida. Southern Medical J. 1992; 85: 442-3.

8. Davidovich T, Rimbroth S, Chazan B, Colodner R, Markel A. Recurrent septicemia and osteomyelitis caused by Pasteurella multocida in a patient with chronic lymphatic leukemia. Israel Med Assoc J. 2008; 10:653-4.

9. Jordan EF, Nye MB, Luque AE. Successful treatment of Pasteurella multocida meningitis with aztreonam. Scand J Infect Dis. 2007; 39:72-4.

10. Goldstein EJC, Citron DM, Richwald GA. Lack of invitro efficacy of oral forms of certain cephalosporins, erythromycin, and oxacillin against Pasteurella multocida. Antimicrob Agent Chemother. 1988; 32: 213-5.

11. Yokose N, Dan K. Pasteurella multocida sepsis, due to a scratch from a pet cat, in a post-chemotherapy neutropenic patient with non-Hodgkin lymphoma. Intern J Hematology. 2007; 85:146-8.

12. Campos Franco J, López Rodríguez R, Ordóñez Barrosa P, Pérez del Molino ML, González Quintela A. Bacteremias primarias por Pasteurella multocida (in Spanish). Anales Medicina Interna. 2008; 25:374.

13. Athar MK, Karim MS, Mannam S, Ahmed M. Fatal Pasteurella sepsis and hairy-cell leukemia. Am J Hematology. 2003; 72:285.

14. Baker GL, Oddis CV, Medsger TA. Pasteurella multocida polyarticular septic arthritis. J Rheumatology. 1987; 14:355-7.

15. Quentin R, Fignon A, Lansac J, Goudeau A. Pasteurella multocida and female genital carcinoma - a case report to dispute the concept of genital opportunistic pathogen. Eur J Obstetr Gynecol Reproduct Biol. 1991; 
41:243-4.

16. Martinez-Albaladejo M, Alguacil-Garcia G, Gonzalez Pina B, Moreno-Requena J. Neumonia por Pasteurella multocida como presentacion de un linfoma pulmonar primario (in Spanish). Anales Medicina Interna. 1998; 15:52.

17. Tanguay JS, Shrihari N, Warren RE. Pasteurella multocida infection following cisplatin and 5-fluorouracil chemotherapy. Clin Oncol. 2003;15:367.

18. Chun ML, Buekers TE, Sood AK, Sorosky JI. Postoperative wound infection with Pasteurella multocida from a pet cat. Am J Obstetr Gynecol. 2003; 188:1115-6.

19. Stein AA, Fialk MA, Blevins A, Armstrong D. Pasteurella multocida septicemia. JAMA. 1983; 249: 508-9.

20. Felix M, Tallon P, Salavert M, Navarro V, Breton JR, Perez-Belles C, Gobernado M. Bacteremia due to Pasteurella spp.: a rare process in our hospital over the last 8 years. Enfermedades Infecciosas Y Microbiologia Clinica. 2003; 21:334-9.
21. Fernandez CH, Garcia MAM, Cataluna JJS, Aguayo JMG, Sanchez PR. Pasteurella multocida infection of cavitated lung squamous carcinoma. Archivos De Bronconeumologia. 2003; 39:236-8.

22. Westbrook CA, Groopman JE, Golde DW. Hairy-cell leukemia-disease pattern and prognosis. Cancer. 1984; 54:500-6.

23. Khan MI, Chan R. Pasteurella multocida subdural empyema - a case report. Can J Neurol Sci. 1981; 8: 163-5.

24. Heyworth MF, Stainforth JN, Wright R, Graham JM. Pasteurella multocida septicemia associated with chronic liver disease. BMJ. 1975; 4:733-4.

25. Butt TS, Khan A, Ahmad A, Khan MA, Parke A, Hill DR. Pasteurella multocida infectious arthritis with acute gout after a cat bite. J Rheumatology. 1997; 24 : 1649-52.

26. Grehn M, Muller F, Hany A, Meier P. Pasteurella multocida septicemia not associated with primary liver disease. Eur J Clin Microbiol Infect Dis. 1984; 3:258-60. 